\title{
Male Gender is independently associated with pulmonary tuberculosis among sputum and non-sputum producers people with presumptive tuberculosis in Southwestern Uganda
}

Yap Boum II ${ }^{1,3^{*}}$, Daniel Atwine ${ }^{1}$, Patrick Orikiriza', Justus Assimwe ${ }^{4}$, Anne-Laure Page ${ }^{2}$, Juliet Mwanga-Amumpaire ${ }^{1,3}$ and Maryline Bonnet ${ }^{2}$

\begin{abstract}
Background: Little is known about the association between gender and risk of TB infection. We sought to assess the impact of gender on TB prevalence among people with presumptive tuberculosis at a regional referral hospital in a high TB and HIV prevalence setting.

Methods: We analyzed data from two diagnostic TB studies conducted in rural, southwestern Uganda. People with presumptive tuberculosis were evaluated by chest X-ray, fluorescence microscopy, TB culture, and HIV testing. Our primary outcome of interest was TB infection, as defined by a positive TB culture. Our primary explanatory variable of interest was gender. We fit univariable and multivariable logistic regression models to investigate associations between TB infection and gender, before and after adjusting or possible confounding factors, including ability to produce sputum, age and residence.

Results: Between April 2010 and September 2012, 863 people with presumptive tuberculosis (PWPTB) were enrolled in the two studies at Mbarara Regional Referral Hospital (MRRH) in Uganda. Among them 664 (76.9\%) were able to produce sputum. X-ray was suggestive of TB for $258(66.5 \%)$ of males and $175(44.8 \%)$ of female $(p<0.001)$. using microscopy 84 (20\%) of males and $48(10.9 \%)$ of females were diagnosed with TB $(p<0.001)$ while 122 (30.3\%) of males and $76(18.4 \%)$ of females were diagnosed with TB $(p<0.001)$ using TB culture. In multivariable logistic regression models, the odds of having TB was higher in males than females (AOR 2.2 (1.56-3.18 95\% $\left.\mathrm{Cl}^{\circ}, \mathrm{P}<0.001\right)$, after adjustment for age, HIV status, ability to produce sputum, and residence.

Conclusion: In Southwestern Uganda, TB prevalence is higher among male than female people with presumptive TB. The increased risk of TB among males is independent of other TB risk factors. These findings emphasize the need for gender-focused interventions aimed at reducing TB transmission.
\end{abstract}

Keywords: Sex, HIV, Risk factors, String test, Sputum induction

\footnotetext{
* Correspondence: yap.boum@epicentre.msf.org

${ }^{1}$ Epicentre Mbarara, Mbarara, Uganda

${ }^{3}$ Mbarara University of Science and Technology, Mbarara, Uganda

Full list of author information is available at the end of the article
} 


\section{Background}

The World Health Organization (WHO) stated that the global male to female prevalence ratio of tuberculosis (TB) is 1.85 [1]. This gender imbalance increases with age and may have implications in the management of TB [1]. While the causes of gender imbalance in TB diagnosis remain unclear and vary among countries, most published studies on the association between gender and TB have not assessed different possible contributing factors in the same setting. To our knowledge, no studies have examined gender differences in non-sputum-producers, mainly HIV infected patients, to assess the effect of gender on TB diagnosis in this population. Moreover, there are no published data on TB prevalence by gender in Southwestern Uganda, a high TB and HIV prevalence setting $[2,3]$.

We determined the prevalence of culture confirmed tuberculosis among men and women presenting to a regional referral hospital in southwestern Ugandan with presumptive tuberculosis (PWPTB) and we assessed the effect of gender on the diagnosis of TB.

\section{Methods}

\section{Study design}

We conducted a secondary analysis of data collected in two cross-sectional diagnostic studies: 1) a study that evaluated the performance of colorimetric culture methods for diagnosis of tuberculosis [4] ("colorimetric study") and 2) a study that assessed the detection yield of alternative specimen collection methods (the "string test study") in patients unable to produce sputum [5].

\section{Participants}

Both studies consecutively recruited participants from the outpatient department (OPD) and the HIV clinic (Immune Suppression Syndrome [ISS] Clinic) at the Mbarara Regional Referral Hospital (MRRH). Patients were eligible if they reported a cough lasting more than two weeks and were older than 15 years of age. Patients able to produce at least $1 \mathrm{~mL}$ of sputum were enrolled in the colorimetric study and patients with less than $1 \mathrm{~mL}$ of sputum or who were unable to produce sputum spontaneously were included in the string test study. In addition, patients without cough but with unexplained weight loss, fever or recent chest $\mathrm{x}$-ray showing radiological features compatible with PTB and who could not produce sputum were eligible for participation in the string test study. Participants were excluded if they had more than seven days of anti-TB treatment prior to enrollment and/or if they were too sick to comply with the sputum induction procedure of the string test study.

\section{Study procedures}

Socio-demographic characteristics specifically age, gender and residence, were collected using a standardized questionnaire which was administered in the local language. Participants underwent a physical examination by a clinical officer to record data on duration and nature of signs and symptoms. Participants were tested for HIV using the algorithm recommended by the Ugandan Ministry of Health STD/AIDS Control Program [4]. Finally, all participants had an antero-posterior chest Xray. Chest $\mathrm{x}$-ray images were interpreted by study clinicians according to a predetermined pictorial tick sheet with quality control by the hospital radiologist and reported as: "normal", "abnormal, possible TB", or "highly suggestive of TB". For this analysis, we considered the latter as radiographic evidence of TB.

For patients enrolled in the colorimetric study [4], two sputum samples were collected including one at the time of enrollment and a second the following morning. For patients enrolled in the string test study [5], participants swallowed a weighted gelatin capsule filled with 90 $\mathrm{cm}$ of nylon string (Entero-test ${ }^{\circ}$, HDC Corporation, San Jose, California, USA), which was retrieved after 2 hours followed by a sputum induction using 5\% hypertonic saline solution nebulized for a maximum of 20 minutes. Two samples were collected during two consecutive days.

Each collected specimen was processed by auramine LED-fluorescence microscopy (on fresh sputum and sediment sample after string test and sputum induction) [4] and TB culture, using both Lowenstein-Jensen (LJ) tubes and a manual mycobacterium growth indicator tube (MGIT) (Becton Dickinson, Franklin Lakes, New Jersey, USA) [4,5]. Observation of acid-fast bacilli (AFB) under the fluorescent microscope was considered a positive smear result, and observation of growth suggestive of $\mathrm{TB}$ on/in TB selective media and confirmed by biochemical/serological tests was considered a positive culture result.

\section{Statistical analysis}

Patient,' specimen characteristics, and test results were summarized by gender, and compared using chi-square test for categorical variables and t-test or rank-sum nonparametric tests for continuous variables. We defined our primary outcome of interest as a culture diagnosis of TB. Our primary explanatory variable of interest was gender. Secondary predictors included age, HIV serostatus, productive versus non-productive cough, urban versus rural residence, presence or absence of a chest $\mathrm{x}$-ray highly suggestive of TB, sputum volume (greater than or less than $1 \mathrm{~mL}$ [6], and macroscopic specimen appearance (mucopurulent/purulent or mucoid/mucosalivary/ salivary/bloody versus not). We fitted univariable and 
multivariable logistic regression models to assess for relationships between gender and our outcome of interest, before and after adjustment for confounding variables.

Data was analyzed with STATA, version 11 (StataCorp, College Station, Texas, USA). A confidence level of $95 \%$ was deemed as statistically significant.

\section{Ethical approval}

Both study protocols were approved by the CPP Ile de France, the Mbarara University Faculty of Medicine Research and Ethics Committee and Institutional Review Committee and the Uganda National Council for Science and Technology. All participants gave written informed consent.

\section{Results}

\section{Participant's enrollment and characteristics}

A total of 863 participants were enrolled in the two studies April 2010 and September 2012, and were included in this analyses. 664 participants $(76.9 \%)$ were enrolled in the colorimetric study and 199 (23.1\%) in the string study (Figure 1).

We enrolled 441 (51\%) female participants. Female PWPTB were younger than males, had a higher prevalence of HIV sero-positivity and were less likely to have chest X-ray results suggestive of pulmonary TB than males (Table 1). There were no differences in other socio-demographic or clinical characteristics by gender.

Specimens' characteristics, microscopy and culture results Sputum specimen characteristics were similar between males and females (Table 1). Significantly more males than females had positive TB microscopy (84/421 [20\%] versus $48 / 441$ [11\%], $P<0.001$, Table 2 ).

\section{Outcomes}

Males were more likely to have a positive AFB sputum smear, and more likely to have high grade $(2+/ 3+)$ smear results (Table 2, Figures 2 and 3). Males were also more likely to have a positive TB culture (30.3\% versus $18.4 \%$, $P=0.001$, Table 2). This was true both for those who produced sputum and those who could not produce sputum. Of the participants with smear-negative results, the males were more likely to have culture positive TB (males: $40 / 318,12.6 \%$, versus females: $29 / 367$, $7.9 \% ; P=0.043)$.

In univariable models gender, age, X-ray and microscopy were associated with a diagnosis of TB by culture. In multivariable models, gender remained significant (AOR 2.2 95\%CI [1.56-3.18]). We also found independent associations with a TB diagnosis for age (Table 3).

\section{Discussion}

In a prospective cohort of people with presumptive tuberculosis at a regional hospital in rural Uganda, male people with presumptive tuberculosis were twice as likely to be diagnosed with culture positive TB compared to females. Although the proportion of males and females seeking care was similar, males represented the majority of culture confirmed TB patients irrespective of their ability to produce sputum, the sputum volume and quality and the HIV status.

Our data are in agreement with data from other settings, which also demonstrate a higher proportion of males with TB culture confirmed compared to women and a higher prevalence of TB in young women [7-11].

TB prevalence in males versus females varies widely among countries, and in some areas prevalence is higher among women. In Pakistan, the majority of TB patients across all age groups are females [12], and in Peru a higher prevalence of TB was found in women of reproductive age compared to males in the same age range

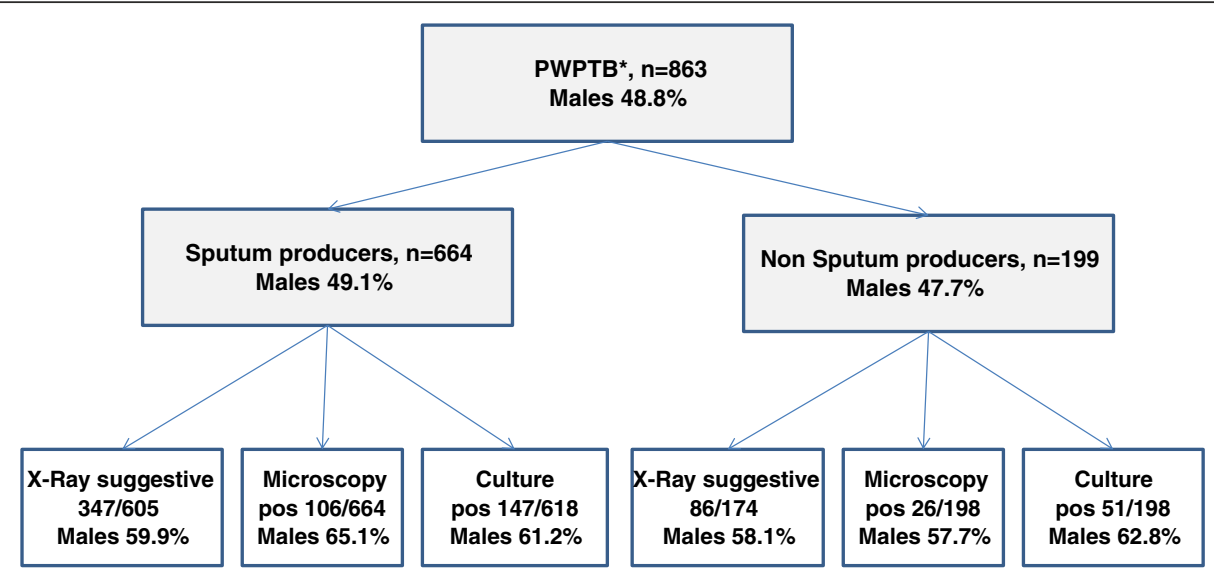

Figure 1 Study profile (*PWPTB patient with presumptive tuberculosis). Number of patients enrolled in the study, results by diagnosis tools with the proportion of positive results among the general population and the percentage of males in the patients with a positive result. 
Table 1 Patient characteristics and by gender

\begin{tabular}{|c|c|c|c|c|}
\hline Variables & Total n (\%) & Male n (\%) & Female $\mathrm{n}(\%)$ & $P$ value \\
\hline Study & $863(100.0)$ & $421(48.8)$ & $442(51.2)$ & 0.737 \\
\hline Sputum producers & $664(76.9)$ & $326(49.1)$ & $338(50.9)$ & \\
\hline Non sputum producers & $199(23.1)$ & $95(47.7)$ & $104(52.3)$ & \\
\hline \multirow[t]{2}{*}{ Age in years $(\mathrm{N}=852)$} & mean (SD) & mean (SD) & mean (SD) & \\
\hline & $39.7(13.9)$ & $41.2(14.3)$ & $38.2(13.4)$ & 0.001 \\
\hline Age categories in years $(\mathrm{N}=852)$ & Total n (\%) & Male $\mathrm{n}(\%)$ & Female $\mathbf{n}(\%)$ & 0.005 \\
\hline$<25$ & $102(12.0)$ & $49(48.0)$ & $53(52.0)$ & \\
\hline $25-35$ & $269(31.6)$ & $113(42.0)$ & $156(58.0)$ & \\
\hline $36-45$ & $241(28.3)$ & $119(49.4)$ & $122(50.6)$ & \\
\hline$>45$ & $240(28.2)$ & $139(57.9)$ & $101(42.1)$ & \\
\hline HIV positive, $\mathrm{N}=820(\%)$ & & & & $<0.001$ \\
\hline Negative & $345(42.1)$ & $196(56.8)$ & $149(43.2)$ & \\
\hline Positive & $475(57.9)$ & $207(43.6)$ & $268(56.4)$ & \\
\hline Residence $(\mathrm{N}=860)$ & & & & 0.411 \\
\hline Urban & $427(49.6)$ & $203(47.5)$ & $224(52.5)$ & \\
\hline Rural & $433(50.4)$ & $218(50.6)$ & $215(49.4)$ & \\
\hline X-ray ,N = 779 (\%) & & & & $<0.001$ \\
\hline Suggestive of TB & $433(55.6)$ & $258(66.5)$ & $175(44.8)$ & \\
\hline Non suggestive of TB & $346(44.4)$ & $130(33.5)$ & $216(55.2)$ & \\
\hline
\end{tabular}

[13].These countries are an exception, however. In Africa and Eastern Europe males seem to represent the majority of adult patients with TB [14-16]. In fact the figures are higher in Eastern Europe than African countries with prevalence as high as $66 \%$ and $88 \%$ in Uzbekistan and Belarus respectively $[7,8]$.

Various reasons have been suggested to explain this gender imbalance in $\mathrm{TB}$ prevalence. Less access to health care for women, and therefore more unreported
$\mathrm{TB}$, has been mentioned in many countries [2,15,17], and the potentially less sensitive screening and diagnosis strategies for women [15] has led to underestimation of $\mathrm{TB}$ in women [15]. Another contributing factor suggested is the higher prevalence of HIV among women in many countries. The paucibacillary nature of pulmonary TB that is common in high HIV prevalence settings leads to an increased proportion of microscopy smear negative results. Therefore any gender disparities in HIV

Table 2 Specimens' characteristics and laboratory results by gender and study

\begin{tabular}{|c|c|c|c|c|}
\hline Colorimetric study (sputum producers) & Total n (\%) & Male $\mathrm{n}(\%)$ & Female $\mathrm{n}(\%)$ & $\mathrm{p}$ \\
\hline Sputum volume $(n=689)$ More than $1 \mathrm{ml}$ & $672(97.5)$ & $331(97.6)$ & $341(97.4)$ & 0.858 \\
\hline Sputum Quality $(n=681)$ Purulent-mucopurulent & $223(32.7)$ & $117(35.2)$ & $106(30.4)$ & 0.176 \\
\hline Microscopy $(\mathrm{N}=664)$ Positive & $106(16.0)$ & $69(21.2)$ & $37(10.9)$ & $<0.001$ \\
\hline TB Culture $(\mathrm{N}=618)$ Positive & $147(23.8)$ & $90(29.3)$ & $57(18.3)$ & 0.001 \\
\hline Xray $(\mathrm{N}=605)$ suggestive & $347(57.4)$ & $208(68.4)$ & $139(46.2)$ & $<0.001$ \\
\hline String test and sputum induction study (non sputum producers) & Total $n(\%)$ & Male n (\%) & Female $\mathrm{n}(\%)$ & $\mathrm{p}$ \\
\hline Microscopy $(\mathrm{N}=198)$ Positive & $26(13.1)$ & $15(15.8)$ & $11(10.7)$ & 0.288 \\
\hline TB Culture $(\mathrm{N}=198)$ Positive & $51(25.7)$ & $32(33.7)$ & 19 (18.5) & 0.014 \\
\hline Xray $(N=174)$ suggestive & $86(49.4)$ & $50(59.5)$ & $36(40.0)$ & 0.010 \\
\hline Both studies & Total n (\%) & Male $\mathrm{n}(\%)$ & Female $\mathrm{n}(\%)$ & $\mathrm{p}$ \\
\hline Microscopy $(\mathrm{N}=862)$ Positive & $132(15.3)$ & $84(20.0)$ & $48(10.9)$ & $<0.001$ \\
\hline TB Culture $(\mathrm{N}=816)$ Positive & $198(24.3)$ & $122(30.3)$ & $76(18.4)$ & $<0.001$ \\
\hline Xray $(\mathrm{N}=779)$ suggestive & $433(55.6)$ & $258(66.5)$ & $175(44.8)$ & $<0.001$ \\
\hline
\end{tabular}




\section{TB Diagnosis by Gender}

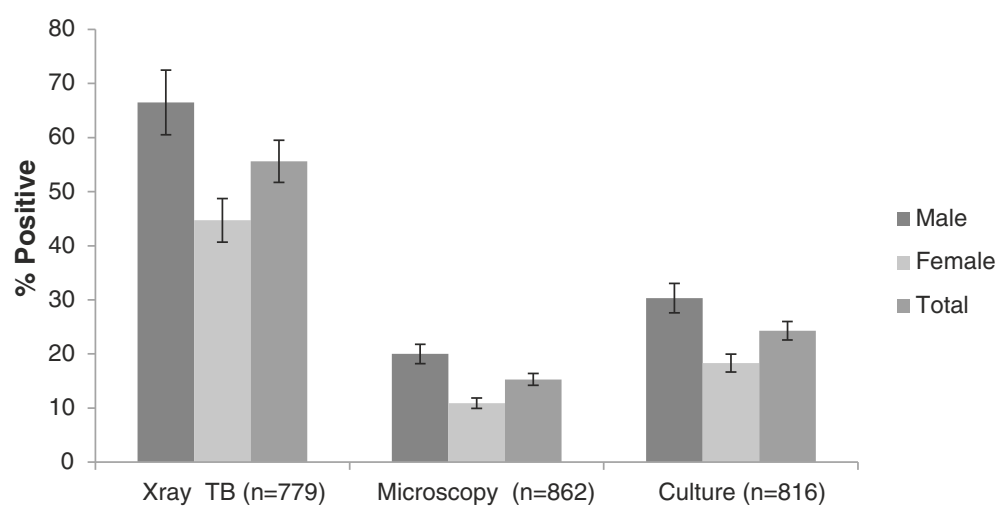

Figure 2 Proportion of X-ray suggestive of TB and TB positive results by microscopy and culture stratified by gender. The right column represents the proportion of male, the middle column represent the proportion of female and the left column the proportion of both in the general population.

prevalence setting are likely to be reflected in TB detection because of the association of HIV with a reduced performance of TB diagnostic tests, especially microscopy [18]. Reliance on microscopy for diagnosing TB in most resource-limited settings could underestimate the TB burden in women who are more likely to be HIV infected than men and typically produce a lower quality of sputum specimen [18]. Other explanations such as social behaviors among men and the difference between male and female susceptibility to TB have also been mentioned $[19,20]$.

It is unlikely that test characteristics could explain the difference we found in TB detection by gender. We used highly sensitive methods for diagnosing pulmonary TB based on the combination of solid and liquid culture methods [21] and on the use of alternative specimen collection methods when patients were unable to produce sputum. Therefore, it is very unlikely that the difference of prevalence of TB between men and women is due to under TB diagnosis in women. The use of culture increased the detection of $\mathrm{TB}$ as described by Lawson et $a l$. in Nigeria [16], who reported similar performance of culture among males and females [16]. In agreement with previous studies women tend to have less smearpositive pulmonary $\mathrm{TB}$ than men. The association between the quality of sputum specimen and the performance of smear-microscopy is well known and could explain partially the gender imbalance among smear positive TB patients, because men tend to produce a better sputum than women [18]. However, in contrast to other studies, in our study there was no difference in volume or quality of sputum specimen between men and women. Moreover the proportion of men having $\mathrm{TB}$ remains significantly higher than

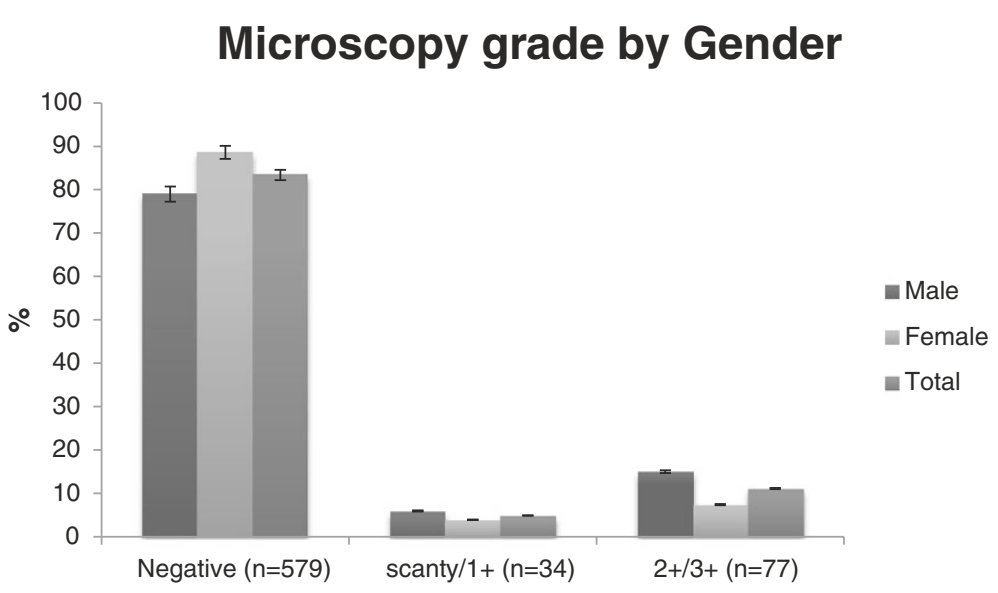

Figure 3 Proportions of microscopy results by gender in sputum producers with presumptive TB. The right column represents the proportion of male, the middle column represent the proportion of female and the left column the proportion of both in the general population. 
Table 3 Unadjusted and adjusted odds ratios for the relationship between gender and TB culture positivity ( $N=779$ )

\begin{tabular}{|c|c|c|c|c|}
\hline Characteristics & OR $(95 \% \mathrm{CI})$ & $P$ value & AOR $(95 \% \mathrm{Cl})$ & $\mathrm{P}$ value \\
\hline Gender & & $<0.001$ & & $<0.001$ \\
\hline Female & REF & & REF & \\
\hline Male & $1.9(1.40-2.69)$ & & $2.2(1.56-3.18)$ & \\
\hline Age (categories in years) & & $<0.001$ & & $<0.001$ \\
\hline$>45$ & REF & & REF & \\
\hline $36-45$ & $1.8(1.09-2.85)$ & & $1.88(1.13-3.12)$ & \\
\hline $25-35$ & $2.2(1.40-3.52)$ & & $2.5(1.56-4.15)$ & \\
\hline$<25$ & $3.3(1.90-5.71)$ & & $3.4(1.90-6.11)$ & \\
\hline \multicolumn{5}{|l|}{ X-Ray } \\
\hline Non Suggestive of TB & REF & & & \\
\hline Suggestive of TB & $5.0(3.29-7.57)$ & 0.001 & & \\
\hline \multicolumn{5}{|l|}{ Microscopy } \\
\hline Negative & REF & & & \\
\hline Positive & $575(139.3-2378.9)$ & $<0.001$ & & \\
\hline Type of cough & & 0.5751 & & \\
\hline Non-productive & REF & & & \\
\hline Productive & $1.0(0.77-1.61)$ & & & \\
\hline HIV status & & 0.1914 & & \\
\hline Negative & REF & & & \\
\hline Positive & $0.8(0.57-1.12)$ & & & \\
\hline Residence & & 0.977 & & \\
\hline Urban & REF & & & \\
\hline Rural & $1.0(0.73-1.38)$ & & & \\
\hline
\end{tabular}

females in non-sputum producers $(33.7 \%$ Vs $18.4 \%$ respectively, $\mathrm{p}=0.014)$.

It is likely that differences in health seeking behaviors are at least partially responsible for the gender-based difference in TB detection we demonstrated. While we did not find a difference in district of residence (as a surrogate of distance to health center), many factors can explain differences in access to health care between men and women, including stigmatization and social factors were not assessed in our study. It has been observed in several settings that women have better health-seeking behaviors compared to men and that men have more advanced symptoms by the time they seek healthcare [22,23]. Similar differences in HIV serostatus, presentation to HIV care, HIV-related mortality have been found by gender [24]. This is likely to be the same in our setting. Indeed, the bacterial load and the proportion of suggestive chest X-ray were significantly higher among men than women, which suggests a late stage of the disease most likely due to late presentation for health care services [18]. Moreover males represent $85 \%$ of patients admitted in the TB ward of Mbarara Hospital, where patients are admitted at a late stage of the disease and require higher level of care, hence confirming our finding (Unpublished data).
Unexpectedly, HIV infection was not associated with TB culture positive results, yet TB is more prevalent among HIV-positive people [8] and MTB detection is known to be less sensitive in HIV infected patients than the HIV non infected [19]. The association between gender and culture results was the same in HIV infected and non-infected patients. Similar findings were described by Lawson et al. in Nigeria [16] with smear microscopy among women. However, in their study, HIVinfected males were less likely to have positive smears than HIV-negative males.

The study has some limitations. First, it focused on patients referred to a regional referral hospital, which might not reflect the same population of people who access health care in rural areas, and might reflect care for those who have been referred on after failure of local therapy or diagnostics. Indeed, an important proportion of the population of Southwestern Uganda does not reach the regional hospital and seeks care in peripheral health centers. Therefore, a higher proportion of women with TB could be potentially diagnosed in more remote facilities. Nevertheless, data from all health centers of the region suggest that this is not the case: males represented $65 \%$ of the 4438 TB cases detected in the 
Southwestern region during the period of our study, which is similar to the proportion of male among culture positive patients in the study (61.6\%). (Asiimwe Justus, personal communication). Secondly, the study did not include any information to assess health seeking behavior and access to health services between men and women as potential factors that could explain differences we found.

\section{Conclusion}

In Southwestern Uganda, we found significantly higher odds of a diagnosis of TB among male people with presumptive tuberculosis. This difference was independent of age, HIV-serostatus, and residence. The higher prevalence of TB in men attending the TB clinic is not due to under-diagnosis in women and seems to be related to delayed diagnosis of TB in men, most likely due to late presentation. These findings emphasize the need for research on understanding social and potential biological determinants of higher prevalence of TB in men and to identify the barriers faced by men in seeking TB healthcare in the early stage of TB. Gender based interventions, such as TB health education targeting men or active screening of TB in men should be pursued. Our data also highlight the need for more targeted interventions in young women, who are at high risk of transmitting $\mathrm{TB}$ to children and, re-emphasize the need for $\mathrm{TB}$ contact tracing.

\section{Competing interests}

The authors declare that they have no competing interests.

\section{Authors' contributions}

YB JMA conceived the study, and participated in its design and coordination and drafted the manuscript. YB AD performed the statistical analysis and drafted the manuscript. MB AJ PO ALP participated in statistical analysis and helped to draft the manuscript. All authors contributed to the writing of the manuscript. All authors read and approved the final manuscript.

\section{Acknowledgements \\ We would like to thank the patients who have participated in the two studies used to perform this analysis and the Mbarara Regional Referral Hospital for allowing Epicentre team to carry out these studies. We are also grateful to Yolanda Muller, Patricia Kahn, to FHI 360's Mario Chen, Laura Philips, Kenneth Schultz and Suzanne Fischer for their support in the preparation of this manuscript. We thank Victoria Katawera and Mark Siedner, for their critical review of the manuscript. \\ Author details \\ ${ }^{1}$ Epicentre Mbarara, Mbarara, Uganda. ${ }^{2}$ Epicentre, Paris, France. ${ }^{3}$ Mbarara University of Science and Technology, Mbarara, Uganda. ${ }^{4}$ Uganda National Tuberculosis and Leprosy program, Mbarara, Uganda.}

Received: 20 June 2014 Accepted: 18 November 2014

Published online: 10 December 2014

\section{References}

1. World Health Organization: Global Tuberculosis Control: A Short Update to the 2009 Report. Geneva, Switzerland: World Health Organization; 2009.

2. Nansera D, Bajunirwe F, Kabakyenga J, Asiimwe PK, Mayanja-Kizza H: Opportunities and barriers for implementation of integrated TB and
HIV care in lower level health units: experiences from a rural western Ugandan district. Afr Health Sci 2010, 10(4):312-319.

3. Nansera D, Bajunirwe F, Elyanu P, Asiimwe C, Amanyire G, Graziano FM: Mortality and loss to follow-up among tuberculosis and HIV co-infected patients in rural southwestern Uganda. Int J Tuberc Lung Dis 2012, 16(10):1371-1376.

4. Boum Y, Orikiriza P, Rojas-Ponce G, Riera-Montes M, Atwine D, Nansumba M, Bazira J, Tuyakira E, DEbeaudrap P, Bonnet M, Page AL: Use of colorimetric culture methods for detection of Mycobacterium tuberculosis complex from sputum samples in resource-limited settings. J Clin Microbiol 2013.

5. Atwine $D$, Boum $Y$, Bonnet $M$, Nansumba M: Diagnostic yield and operational aspects of the "string test" to diagnose tuberculosis in children and adult suspects unable to produce good quality sputum. Mbarara, Uganda. In 43rd Union World Conference on Lung Health, International Union against Tuberculosis and Lung diseases; 2012.

6. Peter JG, Theron G, Pooran A, Thomas J, Pascoe M, Dheda K: Comparison of two methods for acquisition of sputum samples for diagnosis of suspected tuberculosis in smear-negative or sputum-scarce people: a randomised controlled trial. Lancet Respir Med 2013, 1(6):471-478.

7. Diwan VK, Thorson A: Sex, gender, and tuberculosis. Lancet 1999, 353(9157):1000-1001.

8. Thorson A, Hoa NP, Long NH, Allebeck P, Diwan VK: Do women with tuberculosis have a lower likelihood of getting diagnosed? prevalence and case detection of sputum smear positive pulmonary TB, a population-based study from Vietnam. J Clin Epidemiol 2004, 57(4):398-402.

9. Crampin AC, Glynn JR, Floyd S, Malema SS, Mwinuka VK, Ngwira BM, Mwaungulu FD, Warndorff DK, Fine PE: Tuberculosis and gender: exploring the patterns in a case control study in Malawi. Int J Tuberc Lung Dis 2004, 8(2):194-203.

10. Hudelson P: Gender differentials in tuberculosis: the role of socioeconomic and cultural factors. Tuber Lung Dis 1996, 77(5):391-400.

11. Nsubuga P, Johnson JL, Okwera A, Mugerwa RD, Ellner JJ, Whalen CC: Gender and HIV-associated pulmonary tuberculosis: presentation and outcome at one year after beginning antituberculosis treatment in Uganda. BMC Pulm Med 2002, 2:4.

12. Codlin AJ, Khowaja S, Chen Z, Rahbar MH, Qadeer E, Ara I, McCormick JB, Fisher-Hoch SP, Khan AJ: Short report: gender differences in tuberculosis notification in Pakistan. Am J Trop Med Hyg 2011, 85(3):514-517.

13. Onifade DA, Bayer AM, Montoya R, Haro M, Alva J, Franco J, Rosario S, Betty V, Enit V, Ford CM, Acosta CD, Evans CA: Gender-related factors influencing tuberculosis control in shantytowns: a qualitative study. BMC Public Health 2010, 10:381.

14. Boeree MJ, Harries AD, Godschalk P, Demast Q, Upindi B, Mwale A, Nyirenda $T E$, Banerjee A, Salaniponi FM: Gender differences in relation to sputum submission and smear-positive pulmonary tuberculosis in Malawi. Int J Tuberc Lung Dis 2000, 4(9):882-884.

15. Uwizeye CB, De SG, Gilca R, Schwartzman K, Gasana M: Tuberculosis may be underestimated in Rwandan women. Int I Tuberc Lung Dis 2011, 15(6):776-781.

16. Lawson L, Yassin MA, Onuoha AN, Ramsay A, Anderson de Cuevas RR, Theobald S, Davies PDO, Cuevas LE: Yield of smear microscopy and radiological findings of male and female patients with tuberculosis in Abuja, Nigeria. Tuberc Res Treat 2010, 2010:241659.

17. Karim F, Islam MA, Chowdhury AM, Johansson E, Diwan VK: Gender differences in delays in diagnosis and treatment of tuberculosis. Health Policy Plan 2007, 22(5):329-334.

18. Ramsay A, Bonnet M, Gagnidze L, Githui W, Varaine F, Guerin PJ: Sputum, sex and scanty smears: new case definition may reduce sex disparities in smear-positive tuberculosis. Int J Tuberc Lung Dis 2009, 13(5):613-619.

19. Neyrolles O, Quintana-Murci L: Sexual inequality in tuberculosis. PLoS Med 2009, 6(12):e1000199.

20. Uplekar MW, Rangan S, Weiss MG, Ogden J, Borgdorff MW, Hudelson P: Attention to gender issues in tuberculosis control. Int I Tuberc Lung Dis 2001, 5(3):220-224

21. Chihota VN, Grant AD, Fielding K, Ndibongo B, van Zyl A, Muirhead D, Churchyard GJ: Liquid vs. solid culture for tuberculosis: performance and cost in a resource-constrained setting. Int I Tuberc Lung Dis 2010, 14(8):1024-1031.

22. Balasubramanian R, Garg R, Santha T, Gopi PG, Subramani R, Chandrasekaran V, Thomas A, Rajeswari R, Anandakrishnan S, Perumal M, Niruparani C, Sudha G, Jaggarajamma K, Frieden TR, Narayanan PR: Gender 
disparities in tuberculosis: report from a rural DOTS programme in south India. Int J Tuberc Lung Dis 2004, 8(3):323-332.

23. Johansson E, Long NH, Diwan VK, Winkvist A: Gender and tuberculosis control: perspectives on health seeking behaviour among men and women in Vietnam. Health Policy 2000, 52(1):33-51.

24. Cornell M, Schomaker M, Garone DB, Giddy J, Hoffmann CJ, Lessells R Maskew M, Prozesky H, Wood R, Johnson LF, Egger M, Boulle A, Myer L: Gender differences in survival among adult patients starting antiretroviral therapy in South Africa: a multicentre cohort study. PLoS Med 2012, 9(9):e1001304.

doi:10.1186/s12879-014-0638-5

Cite this article as: Boum II et al:: Male Gender is independently associated with pulmonary tuberculosis among sputum and non-sputum producers people with presumptive tuberculosis in Southwestern Uganda. BMC Infectious Diseases 2014 14:638.

\section{Submit your next manuscript to BioMed Central and take full advantage of:}

- Convenient online submission

- Thorough peer review

- No space constraints or color figure charges

- Immediate publication on acceptance

- Inclusion in PubMed, CAS, Scopus and Google Scholar

- Research which is freely available for redistribution 